\title{
Morbimortalidad asociada al Departamento de Neurocirugía del Hospital de Clínicas. Estudio longitudinal retrospectivo durante abril 2017-abril 2019
}

Morbidity and mortality associated to the Neurosurgery Department at the Clinicas University Hospital. Longitudinal retrospective study between April 2017 and 2019 Morbimortalidade associada ao Serviço de Neurocirurgia do Hospital de Clínicas. Estudo longitudinal retrospectivo durante o período abril de 2017-2019

Gonzalo Bertullo', Rodrigo Moragues², Laura Lanning ${ }^{3}$, Víctor Reyes ${ }^{3}$, Agustín Oliveira ${ }^{3}$, Cristian Cardozo ${ }^{3}$, Rodrigo Veiga ${ }^{3}$, Patricia Álvarez ${ }^{3}$

\section{Resumen}

Introducción: la morbimortalidad permite evaluar la calidad asistencial, outcome y comparar diferentes centros asistenciales. Éste es el primer estudio de morbimortalidad en neurocirugía realizado en Uruguay.

Objetivo: determinar la morbimortalidad global y específica en el Departamento de Neurocirugía del Hospital de Clínicas y la asociación entre complicación y morbimortalidad.

Metodología: estudio observacional, descriptivo-analítico, longitudinal, retrospectivo de todos los pacientes $\geq 15$ años que requirieron cirugía entre abril de 2017 y abril de 2019. Los datos se obtuvieron de historias clínicas y se analizaron las siguientes variables: edad, sexo, comorbilidad, clínica, diagnóstico, oportunidad quirúrgica, complicación, tipo de complicación, mortalidad, causa de mortalidad, outcome y tiempo quirúrgico.

Resultados: 477 pacientes fueron intervenidos, 72 complicados. La mortalidad global fue 5,5\% (26/477) y la morbilidad 15\% (72/477). 36\% de los pacientes complicados fallecieron (26/72). La patología vascular fue la morbilidad específica que más se complicó $(20 \%, 14 / 69)$. La infección fue el tipo de complicación más frecuente (46\%, 39/84). La propia evolución de la enfermedad y el terreno causó la muerte del $90 \%$ de los pacientes complicados operados de urgencia (19/21), siendo ésta última factor de riesgo independiente de fallecer $(p=0,018)$. En coordinaciones, la causa de muerte estuvo vinculada al acto quirúrgico (80\%). Hubo asociación entre patología vascular y morbimortalidad $(p=0,015)$ y entre complicación isquémica y morbimortalidad $(p=0,024)$. La presencia de hipertensión endocraneana (HEC) se asoció a un mal resultado $(p=0,003)$.

Conclusiones: los resultados muestran una buena calidad de atención comparado con otros centros. Aún existen aspectos a corregir para reducir la morbimortalidad.

1. Neurocirujano. Asistente Dpto. Neurocirugía, Hospital de Clínicas, Facultad de Medicina, Universidad de la República, Montevideo, Uruguay.

2. Neurocirujano. Prof. Adjto. Dpto. Neurocirugía, Hospital de Clínicas, Facultad de Medicina, Universidad de la República, Montevideo,Uruguay.

3. Estudiante, Facultad de Medicina, Universidad de la República, Montevideo, Uruguay.

Departamento de Neurocirugía, Hospital de Clínicas, Facultad de Medicina, Universidad de la República, Montevideo, Uruguay.

Correspondencia: Dr. Gonzalo Bertullo. Correo electrónico: gonzalobertullo@hotmail.com

No se ha recibido apoyo económico para la realización del trabajo.

No se declaran conflictos de interés

El protocolo de estudio fue aprobado por el Comité de Ética del Hospital de Clínicas

Recibido: 20/12/2020

Aprobado: 15/4/2021

Attribution-NonCommercial 4.0 International (CC BY-NC 4.0) 
Palabras clave: Morbimortalidad

Neurocirugía

Hospitales universitarios

Uruguay

Key words: $\quad$ Morbidity and mortality

Neurosurgery

Hospitals, University

Uruguay

\section{Introducción}

La literatura mundial sobre la morbimortalidad global vinculado al acto neuroquirúrgico es muy limitada.

La mortalidad quirúrgica se define como el número de fallecidos en un período de 30 días posteriores a la intervención quirúrgica ${ }^{(1)}$. Es una medida del outcome utilizada para evaluar la calidad asistencial de una institución o servicio.

La mortalidad global es la proporción de pacientes fallecidos en el total intervenidos (fallecidos/intervenidos), mientras que la mortalidad específica son los pacientes fallecidos por una subespecialidad neuroquirúrgica sobre el total de operados de esa patología específica. No existe una fuente internacional gold standard sobre mortalidad neuroquirúrgica global, hecho que dificulta la comparación interinstitucional ${ }^{(2)}$.

Complicación neuroquirúrgica es cualquier resultado adverso directo no deseado de una cirugía que aparece hasta 30 días luego de la misma ${ }^{(3)}$. El resultado de esta complicación puede determinar morbilidad y/o mortalidad en el paciente.

Morbilidad es la peoría en el estado neurológico del paciente evaluada con la escala modificada de Rankin (mRS), escala universalmente aceptada para medir el grado de discapacidad neurológica y resultados clínicos (figura 1$)^{(4,9)}$. Se define la evolución de los pacientes como: favorable (mRS 0-2); desfavorable (mRS 3-5) y muerte (mRS 6). La morbilidad global es la proporción de número de pacientes que presentaron una complicación en el total de pacientes intervenidos (complicados/intervenidos).

Morbilidad específica representa la proporción de enfermos complicados sobre el total de la patología específica.

Los autores introducen el concepto y definen tres grupos de pacientes para sistematizar el vínculo entre causa y mortalidad.

- Grupo 1 (causa quirúrgica): mortalidad causada directamente por una complicación intraoperatoria.
- Grupo 2 (causa vinculada a la enfermedad de base): mortalidad causada por la propia evolución de la enfermedad neuroquirúrgica de base.

- Grupo 3 (causa médica): mortalidad causada por el terreno, comorbilidad y/o patología médica intercurrente.

Si bien este estudio tiene en esencia una naturaleza retrospectiva, los autores enfatizan que surge del seguimiento prospectivo del $100 \%$ de los casos que comenzó en los ateneos mensuales de morbimortalidad del Dpto. de Neurocirugía a partir del año 2017.

En nuestro medio nunca se realizaron estudios de morbimortalidad en neurocirugía, siendo éste el primer trabajo que nos permite evaluar el estado de situación que tenemos y mejorar los resultados a futuro. Por otra parte, a partir de la revisión bibliográfica exhaustiva no sistematizada realizada, la literatura mundial al respecto es también muy escasa, hecho que impulsa su estudio y análisis, aunque por otro lado dificulta la comparación.

El objetivo general del estudio fue determinar la morbimortalidad global y específica asociada al Dpto. de Neurocirugía del Hospital de Clínicas en el período entre abril de 2017 y abril de 2019.

\section{Metodología}

Se realizó un estudio longitudinal retrospectivo de una serie consecutiva de pacientes con edad $\geq 15$ años, que ingresaron en el Hospital Universitario y se sometieron a tratamiento neuroquirúrgico entre abril de 2017 y abril de 2019.

Se excluyeron aquellos pacientes que recibieron tratamiento endovascular por no corresponder a una cirugía abierta per se, sino a una modalidad de tratamiento intervencionista.

Los datos fueron recolectados directamente de las historias clínicas y registrados en el programa informático EpiData versión 3.1. Se empleó el software IBM SSPS Statistics 20.0 para el análisis estadístico. Para el efecto de las variables independientes en la mortalidad se aplicó un modelo de regresión logística multivariada, tomando como variable dependiente dicotómica la muerte al mes de la intervención y como variables independientes: diagnóstico, tiempo quirúrgico, oportunidad quirúrgica y tipo de complicación. El diagnóstico y tipo de complicación se representaron como 2 grupos de variables individuales dicotómicas a los efectos de analizar cada diagnóstico y complicación independientemente. Se estudió la asociación entre la complicación y la morbilidad mediante dos análisis de $\mathrm{X}^{2}$. Uno se empleó para analizar la asociación entre el tipo de complicación y la morbilidad medida en escala de Rankin, y el otro la asociación entre el diagnóstico y la morbilidad. Las asociaciones se 
Tabla 1. Distribución de la población general según complicaciones y mortalidad por patología

\begin{tabular}{|c|c|c|c|c|c|}
\hline & \multicolumn{5}{|c|}{ Población global n 477} \\
\hline & \multirow[t]{2}{*}{ No complicados } & \multicolumn{2}{|c|}{ Complicados } & \multirow[t]{2}{*}{ Total } & \multirow{2}{*}{$\begin{array}{c}\text { Mortalidad global por } \\
\text { diagnóstico (\%) }\end{array}$} \\
\hline & & Vivos & Fallecidos & & \\
\hline \multicolumn{6}{|l|}{$\operatorname{Edad}(\bar{x})$} \\
\hline Masculino & 44,5 & 41,9 & 42,7 & 43,4 & \\
\hline Femenino & 49,5 & 45,7 & 52,3 & 49 & \\
\hline \multicolumn{6}{|l|}{ Sexo } \\
\hline Masculino & $226(47,4 \%)$ & 29 & $14(9,0 \%)$ & $269(56,4 \%)$ & \\
\hline Femenino & $179(37,5 \%)$ & 17 & $12(6,1 \%)$ & $208(43,6 \%)$ & \\
\hline \multicolumn{6}{|l|}{ Oportunidad } \\
\hline Coordinación & $218(45,7 \%)$ & 31 & $5(7,5 \%)$ & $254(53,2 \%)$ & \\
\hline Urgencia & $187(39,2 \%)$ & 15 & $21(7,5 \%)$ & $223(46,8 \%)$ & \\
\hline \multicolumn{6}{|l|}{ Diagnósticos } \\
\hline Tumoral & $112(23,5 \%)$ & 17 & $5(4,6 \%)$ & $134(28,1 \%)$ & $3,7 \%$ \\
\hline Traumático & $101(21,2 \%)$ & 6 & $5(2,3 \%)$ & $112(23,5 \%)$ & $4,5 \%$ \\
\hline Vascular & $45(9,4 \%)$ & 10 & $14(5,0 \%)$ & $69(14,5 \%)$ & $20,3 \%$ \\
\hline Columna & $59(12,4 \%)$ & 5 & $0(1,0 \%)$ & $64(13,4 \%)$ & $0,0 \%$ \\
\hline Funcional & $15(3,1 \%)$ & 0 & $0(0,0 \%)$ & $15(3,1 \%)$ & $0,0 \%$ \\
\hline Infeccioso & $12(2,5 \%)$ & 0 & $1(0,2 \%)$ & $13(2,7 \%)$ & $7,7 \%$ \\
\hline Nervios periféricos & $9(1,9 \%)$ & 2 & $0(0,4 \%)$ & $11(2,3 \%)$ & $0,0 \%$ \\
\hline Otros & $52(10,9 \%)$ & 6 & $1(1,5 \%)$ & $59(12,4 \%)$ & $1,7 \%$ \\
\hline
\end{tabular}

consideraron significativas a partir de un nivel de valor $p$ $\leq 0,05$.

\section{Resultados}

\section{Morbimortalidad global}

Se llevaron a cabo un total de 571 intervenciones quirúrgicas en 477 pacientes. La morbilidad global del servicio fue de $15 \%$ (72/477). El total de los 72 pacientes complicados requirieron de 160 intervenciones, o sea, las 88 restantes correspondieron a reintervenciones $(55 \%)$.

La media de edad fue de 49 años en la mujer y 44 años en hombres. La distribución por sexo fue de $44 \%$ para el femenino y $56 \%$ masculino. Según la oportunidad quirúrgica el 53\% fueron cirugías coordinadas y $47 \%$ urgencias (tabla 1).
0 Sin sintomas.

1 Sin incapacidad significativa. A pesar de síntomas realiza actividades cotidianas.

2 Incapacidad leve. Incapaz de realizar las actividades previas pero capaz de hacer algunas actividades sin asistencia.

3 Incapacidad moderada. Requiere alguna ayuda pero es capaz de caminar sin ayuda.

4 Incapacidad moderada severa. Incapacidad de caminar sin ayuda e incapaz de realizar sus necesidades corporales $\sin$ ayuda.

5 Incapacidad severa. Confinado a cama, incontinente y requiere cuidado constante de enfermería 6 Defunción.

Figura 1. Escala de Rankin modificada (mRS). 


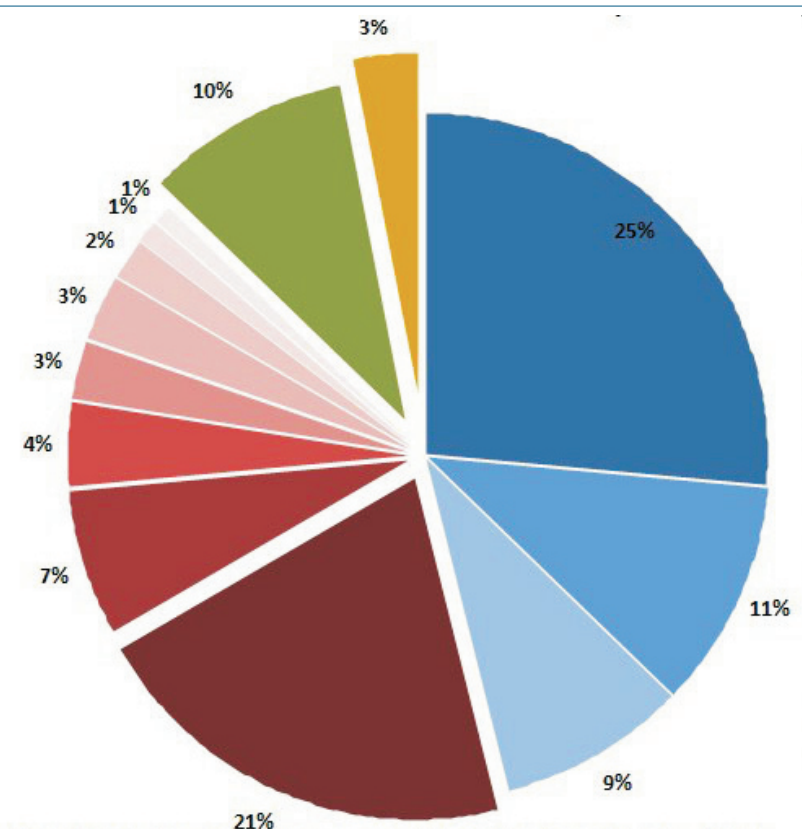

Tabaquismo

Consumo de alcohol

Otras sustancias

Hipertensión arterial

Diabetes Mellitus

Hipotiroidismo

Anticoagulación

Patología respiratoria

Cardiopatía

Dislipemia

Inmunocompromiso

- Patología oncológica

Otras

Gama de azul: patología de consumo. Gama de rojo: patología médica

Gama de verde: patología oncológica. Amarillo: otras

Figura 2. Comorbilidades de pacientes complicados.

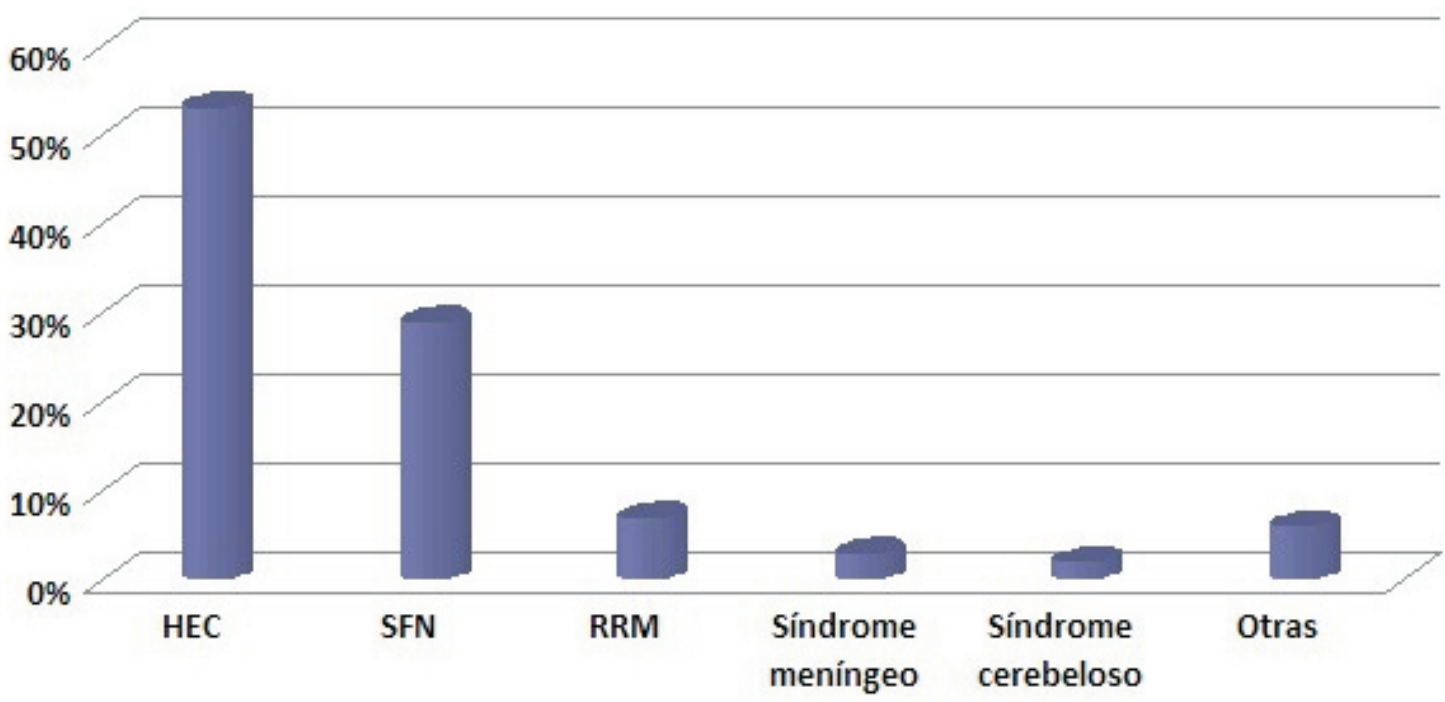

Figura 3. Presentación clínica al ingreso de los pacientes complicados. Nótese la alta prevalencia de hipertensión endocraneana. HEC: hipertensión endocraneana; RRM: raqui-radículo-medular; SFN: síndrome focal neurológico.

La media de edad en los pacientes complicados fue de 45 años, $40 \%$ (29) fueron del sexo femenino y $60 \%$ (43) masculino. De éstos, la mitad se operó de urgencia y la otra mitad de coordinación (tabla 1).

La comorbilidad más frecuente fue la patología de consumo (46\%) representado por el tabaquismo en el 57\%. La hipertensión arterial (HTA), la diabetes melli- tus tipo II (DM II) y el hipotiroidismo fueron las patologías médicas más frecuentes, seguidas por las oncológicas (figura 2).

La forma de presentación clínica más frecuente al ingreso fue el síndrome de hipertensión endocraneana (HEC) en un 53\%, dado por cefalea $(69 \%)$ y depresión de conciencia (57\%) (figura 3). El 51\% (37/72) de los 
Tabla 2. Distribución total de pacientes complicados según oportunidad quirúrgica de urgencia y evolución postquirúrgica evaluada mediante mRS.

\begin{tabular}{|c|c|c|c|c|}
\hline & \multicolumn{4}{|c|}{ Urgencia $n=36$} \\
\hline & $m R S 0-2$ & $m R S 3-5$ & $m R S 6$ & Total \\
\hline \multicolumn{5}{|l|}{ Sexo } \\
\hline Masculino & $8(22,2 \%)$ & $2(5,5 \%)$ & $11(30,5 \%)$ & $21(55,6 \%)$ \\
\hline Femenino & $3(8,3 \%)$ & $2(5,5 \%)$ & $10(27,7 \%)$ & $15(44,4 \%)$ \\
\hline Total & $11(30,5 \%)$ & $4(11,0 \%)$ & $21(58,3 \%)$ & $36(100 \%)$ \\
\hline \multicolumn{5}{|l|}{ Edad } \\
\hline $15-29$ & $6(16,7 \%)$ & 0 & $4(11,1 \%)$ & $10(27,8 \%)$ \\
\hline $30-44$ & 0 & $2(5,6 \%)$ & $3(8,3 \%)$ & $5(13,9 \%)$ \\
\hline $45-59$ & $4(11,1 \%)$ & $1(2,8 \%)$ & $10(27,8 \%)$ & $15(41,7 \%)$ \\
\hline $60-76$ & $1(2,8 \%)$ & $1(2,8 \%)$ & $4(11,1 \%)$ & $6(16,7 \%)$ \\
\hline Total & $11(30,6 \%)$ & $4(11,1 \%)$ & $21(58,3 \%)$ & $36(100 \%)$ \\
\hline \multicolumn{5}{|l|}{ Diagnóstico } \\
\hline Traumático & $5(13,9 \%)$ & $1(2,8 \%)$ & $5(13,9 \%)$ & $11(30,6 \%)$ \\
\hline Vascular & $4(11,1 \%)$ & $3(8,3 \%)$ & $13(36,1 \%)$ & $20(55,6 \%)$ \\
\hline Tumoral & 0 & 0 & $1(2,8 \%)$ & $1(2,8 \%)$ \\
\hline Columna & 0 & 0 & 0 & 0 \\
\hline Nervios periféricos & 0 & 0 & 0 & 0 \\
\hline Infeccioso & 0 & 0 & $1(2,8 \%)$ & $1(2,8 \%)$ \\
\hline Otros & $2(5,6 \%)$ & 0 & $1(2,8 \%)$ & $3(8,3 \%)$ \\
\hline Total & $11(30,6 \%)$ & $4(11,1 \%)$ & $21(58,3 \%)$ & $36(100 \%)$ \\
\hline \multicolumn{5}{|l|}{ Tipo de complicación } \\
\hline Infecciosa & $9(24,3 \%)$ & $3(8,1 \%)$ & $8(21,6 \%)$ & $20(54,1 \%)$ \\
\hline Hemorrágica & $5(13,5 \%)$ & 0 & $1(2,7 \%)$ & $6(16,2 \%)$ \\
\hline Fístula LCR & $1(2,7 \%)$ & 0 & 0 & $1(2,7 \%)$ \\
\hline Hidrocefalia & $2(5,4 \%)$ & 0 & $1(2,7 \%)$ & $3(8,1 \%)$ \\
\hline Isquémica & 0 & $1(2,7 \%)$ & $2(5,4 \%)$ & $3(8,1 \%)$ \\
\hline Otros & 0 & $1(2,7 \%)$ & $3(8,1 \%)$ & $4(10,8 \%)$ \\
\hline Total & $17(45,9 \%)$ & $5(13,5 \%)$ & $15(40,5 \%)$ & $37(100 \%)$ \\
\hline \multicolumn{5}{|l|}{ Causa de muerte } \\
\hline Quirúrgica (grupo 1) & & & & $2(9,5 \%)$ \\
\hline Enfermedad (grupo 2) & & & & $14(66,7 \%)$ \\
\hline Médica (grupo 3) & & & & $5(23,8 \%)$ \\
\hline Total & & & & $21(100 \%)$ \\
\hline
\end{tabular}


Tabla 3. Distribución total de pacientes complicados según oportunidad quirúrgica de coordinación y evolución postquirúrgica evaluada mediante mRS.

\begin{tabular}{|c|c|c|c|c|}
\hline & \multicolumn{4}{|c|}{ Coordinación $n=36$} \\
\hline & $m R S 0-2$ & $m R S 3-5$ & $m R S 6$ & Total \\
\hline \multicolumn{5}{|l|}{ Sexo } \\
\hline Masculino & $16(44,4 \%)$ & $3(8,3 \%)$ & $4(11,1 \%)$ & $23(63,9 \%)$ \\
\hline Femenino & $10(27,8 \%)$ & $2(5,6 \%)$ & $1(2,8 \%)$ & $13(36,1 \%)$ \\
\hline Total & $26(72,2 \%)$ & $5(13,9 \%)$ & $5(13,9 \%)$ & $36(100 \%)$ \\
\hline \multicolumn{5}{|l|}{ Edad } \\
\hline $15-29$ & $6(16,7 \%)$ & 0 & 0 & $6(16,7 \%)$ \\
\hline $30-44$ & $9(25,0 \%)$ & 0 & $1(2,8 \%)$ & $10(27,8 \%)$ \\
\hline $45-59$ & $5(13,9 \%)$ & $3(8,3 \%)$ & $3(8,3 \%)$ & $11(30,6 \%)$ \\
\hline $60-76$ & $6(16,7 \%)$ & $2(5,6 \%)$ & $1(2,8 \%)$ & $9(25,0 \%)$ \\
\hline Total & $26(72,2 \%)$ & $5(13,9 \%)$ & $5(13,9 \%)$ & $36(100 \%)$ \\
\hline \multicolumn{5}{|l|}{ Diagnóstico } \\
\hline Traumático & 0 & 0 & 0 & 0 \\
\hline Vascular & $3(8,3 \%)$ & 0 & $1(2,8 \%)$ & $4(11,1 \%)$ \\
\hline Tumoral & $13(36,1 \%)$ & $4(11,1 \%)$ & $4(11,1 \%)$ & $21(58,3 \%)$ \\
\hline Columna & $4(11,1 \%)$ & $1(2,8 \%)$ & 0 & $5(13,9 \%)$ \\
\hline Nervios periféricos & $2(5,6 \%)$ & 0 & 0 & $2(5,6 \%)$ \\
\hline Infeccioso & 0 & 0 & 0 & 0 \\
\hline Otros & $4(11,1 \%)$ & 0 & 0 & $4(11,1 \%)$ \\
\hline Total & $26(72,2 \%)$ & $5(13,9 \%)$ & $5(13,9 \%)$ & $36(100 \%)$ \\
\hline \multicolumn{5}{|l|}{ Tipo de complicación } \\
\hline Infecciosa & $16(34,0 \%)$ & $2(4,3 \%)$ & $1(2,1 \%)$ & $19(40,4 \%)$ \\
\hline Hemorrágica & $4(8,5 \%)$ & $2(4,3 \%)$ & $4(8,5 \%)$ & $10(21,3 \%)$ \\
\hline Fístula LCR & $7(14,9 \%)$ & $1(2,1 \%)$ & 0 & $8(17,0 \%)$ \\
\hline Hidrocefalia & $3(6,4 \%)$ & $1(2,1 \%)$ & 0 & $4(8,5 \%)$ \\
\hline Isquémica & 0 & $1(2,1 \%)$ & $1(2,1 \%)$ & $2(4,3 \%)$ \\
\hline Otros & $3(6,4 \%)$ & $1(2,1 \%)$ & 0 & $4(8,5 \%)$ \\
\hline Total & $33(70,2 \%)$ & $8(17,0 \%)$ & $6(12,8 \%)$ & $47(100 \%)$ \\
\hline \multicolumn{5}{|l|}{ Causa de muerte } \\
\hline Quirúrgica (grupo 1) & & & & $4(80,0 \%)$ \\
\hline Enfermedad (grupo 2) & & & & 0 \\
\hline Médica (grupo 3) & & & & $1(20,0 \%)$ \\
\hline Total & & & & $5(100 \%)$ \\
\hline
\end{tabular}




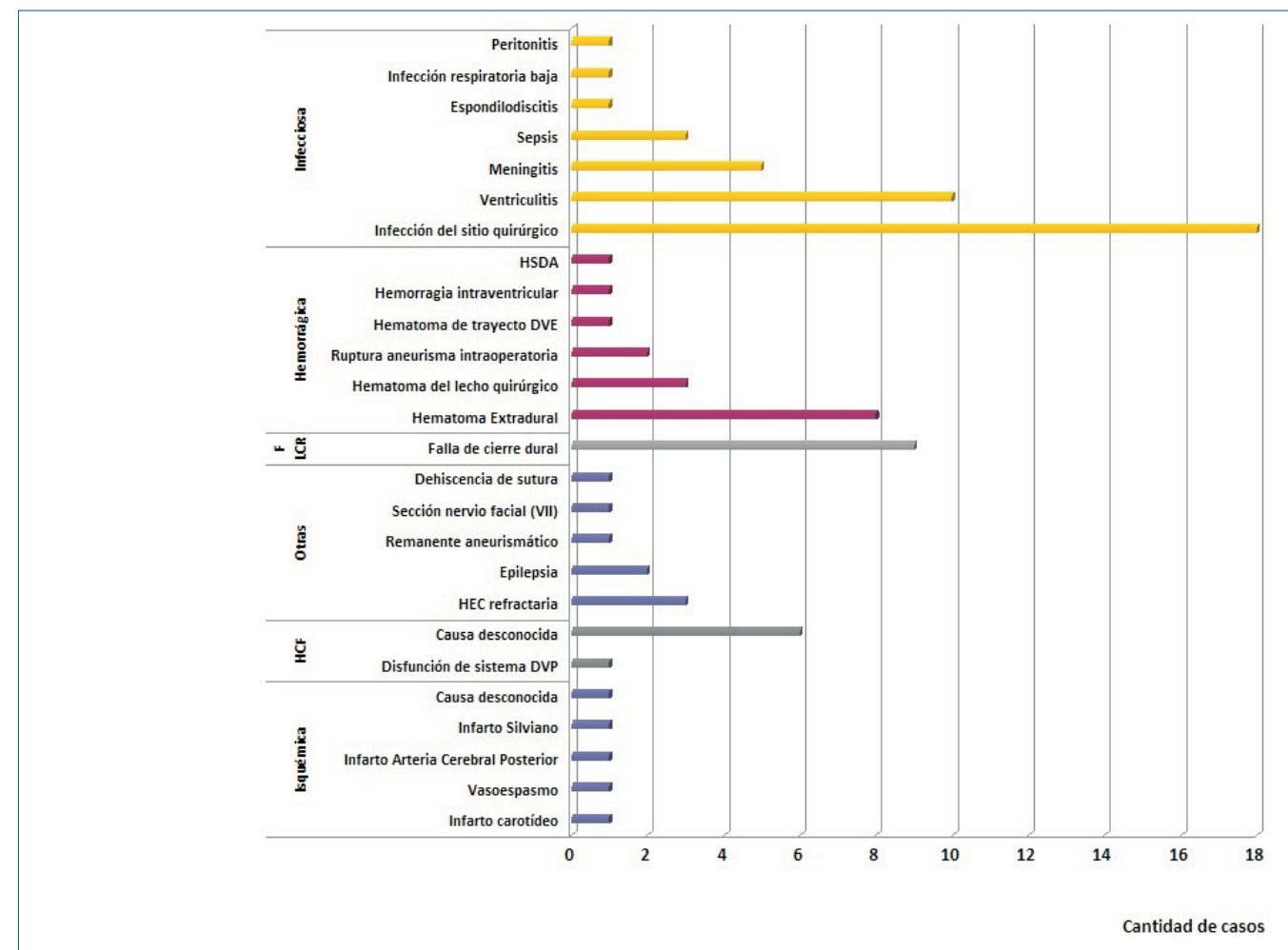

Figura 4. Tipos de complicaciones. Obsérvese la alta frecuencia de complicaciones infecciosas. FLCR: fístula de líquido cefalorraquídeo.

pacientes complicados presentaron una evolución favorable y el $12,5 \%(9 / 72)$ fue desfavorable (mRS 3-5) (tabla 5).

Discriminados por urgencia, $15 \%$ (11/72) tuvo evolución favorable y 5,5\% (4/72) desfavorable (tabla 2). De los coordinados, $36 \%$ (26/72) evolucionaron favorablemente y $7 \%(5 / 72)$ desfavorablemente (tabla 3$)$.

De los 72 pacientes complicados encontramos 84 tipos de complicaciones en total, donde la infección fue el tipo más frecuente en un 46\% (39/84) y dentro de ésta la infección del sitio quirúrgico predominó en un $46 \%$ (18/39). Hubo 10 casos de ventriculitis $(26 \%)$ y 5 casos de meningitis (13\%) (tabla 4 y figura 4$)$.

La causa hemorrágica fue el segundo tipo de complicación en 19\% (16/84), siendo el hematoma extradural (HED) del colgajo el que predominó en la mitad de los casos 50\% (8/16) (tabla 4). El resto se vinculó a hematomas del lecho quirúrgico en tres casos (19\%) y en dos casos a ruptura aneurismática intraoperatoria $(12 \%)$. Hemorragia intraventricular (HIV), hematoma subdural agudo (HSDA) y hematoma en trayecto de derivación ventricular externa (DVE) se observaron una sola vez ( $6 \%$ cada uno). Otros tipos de complicaciones fueron fístula de líquido cefalorraquídeo (FLCR) en un $11 \%$ (9 casos), isquemia $6 \%$ (5 casos) y 7 casos de hidrocefalia
(8\%). El 10\% restante se consignó como "otro tipo de complicaciones", donde la HEC refractaria fue la complicación más diagnosticada (figura 4).

La mortalidad global del servicio fue de 5,5\% (26/477), 54\% sexo masculino y $46 \%$ del sexo femenino, para una media de edad de 47 años. Sólo el 19\% de los fallecidos (5/26) fueron operados de coordinación y $81 \%$ de urgencia (21/26). Esto determina una mortalidad global de coordinación de $1,9 \%$ (5/254) y $9,4 \%$ de urgencia (21/223) (tabla 1). La mortalidad global por patología predominó en lo vascular con $20 \%$ (14/69), seguida por la patología infecciosa $7,7 \%(1 / 13)$, traumática con $4,5 \%$ (5/112), tumoral 3,7\% (5/134). En las patologías de columna, funcionales y de nervios periféricos no hubo muertes (tabla 1). La complicación que llevó a la muerte en los pacientes coordinados fue en un $80 \%(4 / 5)$ de causa quirúrgica (grupo 1) y un $20 \%(1 / 5)$ de causa médica vinculado a sepsis (grupo 3). De los que fallecieron por causa quirúrgica el hematoma del lecho representó $75 \%$ (3/4) y la isquemia por clipado inadvertido de la arteria carótida interna el 25\% (1/4) (tabla 4).

La causa de muerte en los pacientes intervenidos de urgencia mostró un $9 \%(2 / 21)$ por causa quirúrgica (grupo 1$), 67 \%(14 / 21)$ por la propia evolución de su enfer- 
Tabla 4. Distribución total de pacientes complicados según oportunidad quirúrgica y sexo, edad, diagnóstico al ingreso, tipo de complicación y causa de muerte.

\begin{tabular}{|c|c|c|c|}
\hline & Urgencia & Coordinación & Total complicados $(n=72)$ \\
\hline \multicolumn{4}{|l|}{ Sexo } \\
\hline Masculino & $20(55,6 \%)$ & $23(63,9 \%)$ & $43(59,7 \%)$ \\
\hline Femenino & $16(44,4 \%)$ & $13(36,1 \%)$ & $29(40,3 \%)$ \\
\hline Total & $36(100 \%)$ & $36(100 \%)$ & $72(100 \%)$ \\
\hline \multicolumn{4}{|l|}{ Edad } \\
\hline $15-29$ & $10(27,8 \%)$ & $6(16,7 \%)$ & $16(22,2 \%)$ \\
\hline $30-44$ & $5(13,9 \%)$ & $10(27,8 \%)$ & $15(20,8 \%)$ \\
\hline $45-59$ & $15(41,7 \%)$ & $11(30,6 \%)$ & $26(36,1 \%)$ \\
\hline $60-76$ & $6(16,7 \%)$ & $9(25,0 \%)$ & $15(20,8 \%)$ \\
\hline Total & $36(100 \%)$ & $36(100 \%)$ & $72(100 \%)$ \\
\hline \multicolumn{4}{|l|}{ Diagnóstico } \\
\hline Traumático & $11(30,6 \%)$ & 0 & $11(15,3 \%)$ \\
\hline Vascular & $20(55,6 \%)$ & $4(11,1 \%)$ & $24(33,3 \%)$ \\
\hline Tumoral & $1(2,8 \%)$ & $21(58,3 \%)$ & $22(30,6 \%)$ \\
\hline Columna & 0 & $5(13,9 \%)$ & $5(6,9 \%)$ \\
\hline Nervios periféricos & 0 & $2(5,6 \%)$ & $2(2,8 \%)$ \\
\hline Infeccioso & $1(2,8 \%)$ & 0 & $1(1,4 \%)$ \\
\hline Otros & $3(8,3 \%)$ & $4(11,1 \%)$ & $7(9,7 \%)$ \\
\hline Total & $36(100 \%)$ & $36(100 \%)$ & $72(100 \%)$ \\
\hline \multicolumn{4}{|l|}{ Tipo de complicación } \\
\hline Infecciosa & $20(54,1 \%)$ & $19(40,4 \%)$ & $39(46,4 \%)$ \\
\hline Hemorrágica & $6(16,2 \%)$ & $10(21,3 \%)$ & $16(19,0 \%)$ \\
\hline Fístula LCR & $1(2,7 \%)$ & $8(17,0 \%)$ & $9(10,7 \%)$ \\
\hline Hidrocefalia & $3(8,1 \%)$ & $4(8,5 \%)$ & $7(8,3 \%)$ \\
\hline Isquémica & $3(8,1 \%)$ & $2(4,3 \%)$ & $5(6,0 \%)$ \\
\hline Otras & $4(10,8 \%)$ & $4(8,5 \%)$ & $8(9,5 \%)$ \\
\hline Total & $37(100 \%)$ & $47(100 \%)$ & $84(100 \%)$ \\
\hline \multicolumn{4}{|l|}{ Causa de muerte } \\
\hline Quirúrgica (grupo 1) & $2(9,5 \%)$ & $4(80,0 \%)$ & $6(23,1 \%)$ \\
\hline Enfermedad (grupo 2) & $14(66,7 \%)$ & 0 & $14(53,8 \%)$ \\
\hline Médica (grupo 3) & $5(23,8 \%)$ & $1(20,0 \%)$ & $6(23,1 \%)$ \\
\hline Total & $21(100 \%)$ & $5(100 \%)$ & $26(100 \%)$ \\
\hline
\end{tabular}

LCR: líquido cefalorraquídeo. 
medad de base (grupo 2$)$ y $24 \%(5 / 21)$ por causa médica (grupo 3) (ó 5).

En el grupo 1 las causas de muerte fueron HED y ventriculitis.

En el grupo 2 la HEC refractaria representó un \% (6/14), el vasoespasmo $21 \%$ (3/14). La propia evolución de un infarto cerebral maligno, resangrado, hinchazón cerebral, compresión de tronco encefálico y de un glioblastoma representaron cada una el $7 \%$ restante (1/14). En el grupo 3 la totalidad de los casos fueron muerte causada por sepsis de origen respiratorio.

\section{Morbimortalidad específica}

Del análisis por entidad específica de los pacientes complicados y fallecidos, observamos que el diagnóstico de ingreso más frecuente fue vinculado a la patología vascular, accidente cerebro vascular (ACV) en 33\% (24/72), representado por la naturaleza hemorrágica en un $92 \%(22 / 24)$ e isquémica en $8 \%(2 / 24)$ (tabla 5). Dentro del ACV hemorrágico el diagnóstico nosológico que más se complicó fue la hemorragia subaracnoidea espontánea (HSAe) en 73\% (16/22).

El hematoma cerebral lobar fue el segundo diagnóstico que siguió luego de la HSAe, en 14\% (3/22). Se diagnosticaron dos hematomas cerebelosos (9\%), uno de los cuales se presentó en un contexto hipertensivo y el otro en un paciente anticoagulado. Un solo paciente anticoagulado hizo un HSDA (4,5\%).

La etiología complicada más frecuente del ACV hemorrágico fue la causa aneurismática en el 59\% (13/22), malformaciones arteriovenosas (MAV) en 9\% (2/22) y finalmente la administración de anticoagulantes orales y consumo de cocaína en 4,5\% cada uno. En $23 \%(5 / 22)$ no se pudo determinar la causa. De los 2 pacientes diagnosticados con ACV isquémico uno de ellos fue por una disección carotídea, y en el otro no se pudo determinar la causa. Del 56\% global de pacientes complicados con ACV operados de urgencia (20/36), solo el 20\% (4/20) tuvo una evolución favorable, $15 \%$ (3/20) tuvo una evolución desfavorable y $65 \%$ fallecieron (13/20) (tabla 2). En los coordinados el 75\% (3/4) de los casos presentaron una evolución favorable y el caso restante falleció (25\%) (tabla 3).

En cuanto a la mortalidad específica en la patología vascular hubo un $58 \%$ de pacientes fallecidos (14/24), 93\% habían sido operados de urgencia (13/14) y uno de coordinación (7\%) (tabla 5). La media de edad fue de 53 años.

De los pacientes vasculares que fallecieron en la urgencia, el 69\% (9/13) fue debido a la propia evolución de la enfermedad de base (grupo 2). El 15\% fue vinculado a causa quirúrgica (grupo 1) y el 15\% restante de causa médica (grupo 3) (tabla 5). De los cuatro pacien- tes complicados operados de coordinación, el único fallecido correspondió a un infarto carotídeo por "kinking" inadvertido de la arteria carótida interna en clipado de aneurisma comunicante posterior (grupo 1) $(25 \%)$.

La patología tumoral fue la segunda causa de complicación por diagnóstico al ingreso en $31 \%$ de los casos (22/72). El 86\% (19/22) fueron tumores intracraneanos, mientras que el restante $14 \%$ fueron tumores espinales (3/22). Respecto a los tumores intracraneanos el complicado más frecuente fue el glioma de alto grado en $21 \%$ (4/19), seguido del meningioma (16\%, 3/19). Metástasis, glioma de bajo grado, adenoma hipofisario y quiste de inclusión determinaron 2 casos cada uno ( $10,5 \%$ del total) y un caso de neurinoma, remanente tumoral, quiste aracnoideo y carcinoma neuroendocrino de células pequeñas de alto grado (5,2\% cada uno).

Se diagnosticaron 3 tumores espinales, de los cuales 2 fueron metástasis y uno ependimoma. El 66,6\% de los pacientes $(2 / 3)$ tuvieron un desenlace favorable mientras que uno diagnosticado con metástasis espinal con compresión medular evolucionó desfavorablemente $(33,3 \%)$

En los pacientes coordinados por patología tumoral que se complicó (21/22), el 62\% (13/21) evolucionó favorablemente (mRS 0-2), 19\% (4/21) evolucionó desfavorablemente (mRS 3-5) y el restante 19\% (4/21) (mRS 6) falleció (tabla 5).

La mortalidad especifica de la patología tumoral fue de $23 \%(5 / 22)$, de los cuales un $60 \%$ (3/5) fueron por causa quirúrgica (grupo 1) (2 hemorragias y una causa indeterminada), $20 \%(1 / 5)$ por Sepsis (grupo 3$)$ y $20 \%(1 / 5)$ por la propia evolución de la enfermedad de una metástasis (grupo 2), ésta fue la única paciente intervenida de urgencia, los restantes cuatro fueron por coordinación.

La patología traumática fue la entidad que se complicó en tercer lugar, en un 15\% (11/72) y el 100\% de ellos fue intervenidos de urgencia (tabla 5). De éstos, el $82 \%$ (9/11) fue un traumatismo cráneo encefálico (TCE) por siniestro de tránsito y $18 \%$ por herida causada por arma de fuego (HAF) en cráneo (2/11). Dentro de la etiología de los TCE el HSDA se diagnosticó en 29\% (4/14). El hundimiento de cráneo, la hinchazón cerebral y la HAF se presentaron en dos oportunidades (14\% cada uno). Contusión cerebral, contusión cerebelosa, HED y hematoma subdural crónico (HSDC) en una oportunidad (7\% cada uno). El 45\% (5/11) evolucionó favorablemente, 9\% (1/11) evolucionó desfavorablemente y se observó una mortalidad específica de esta patología de $45 \%$ $(5 / 11)$. De los fallecidos, el 60\% (3/5) murieron por causa de la evolución de la propia enfermedad (grupo 2) (HEC refractaria) y los restantes dos pacientes (40\%) ambos debido a sepsis (grupo 3). 


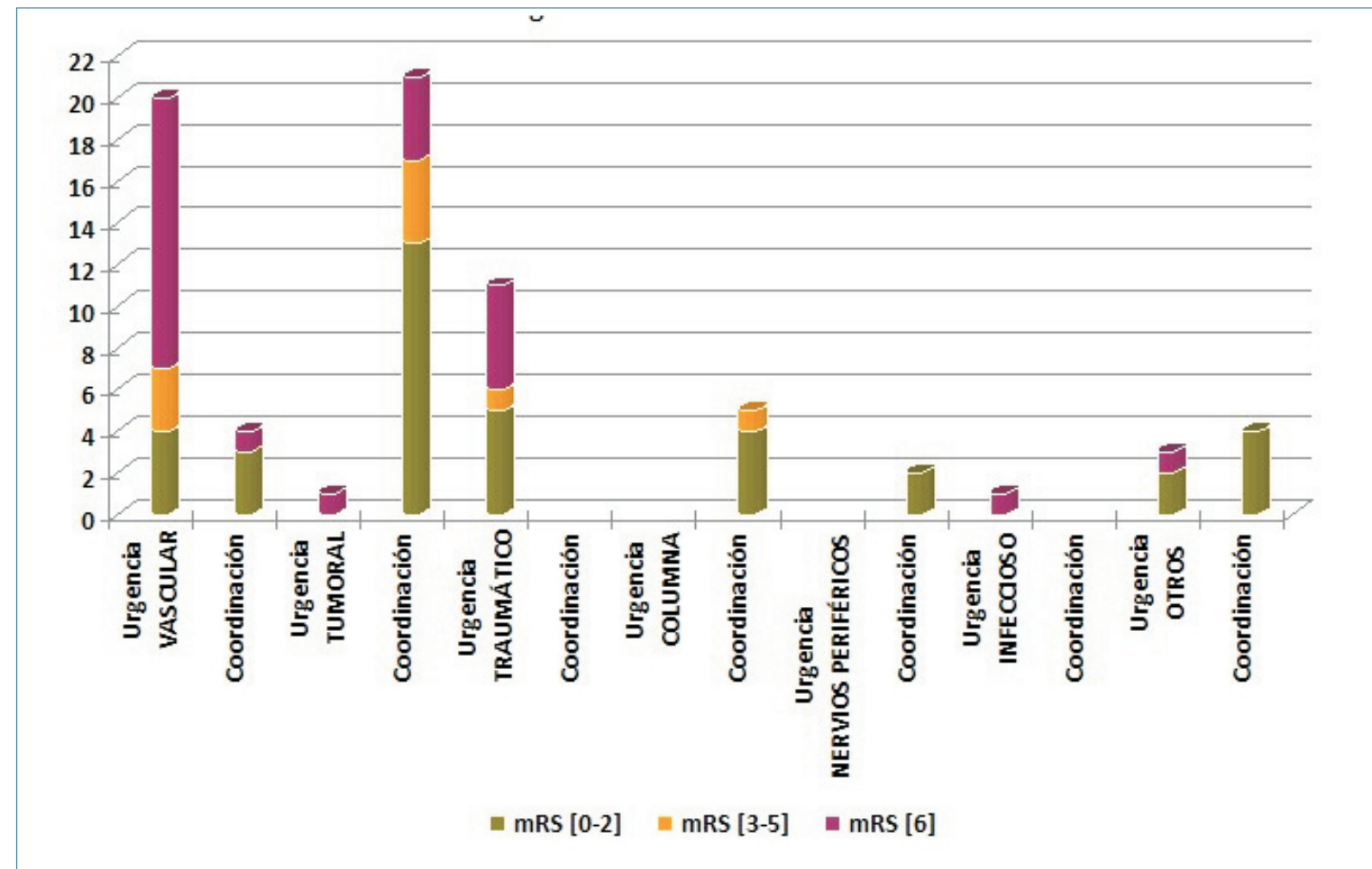

Figura 5. Morbimortalidad específica según patología y oportunidad quirúrgica. Véase la alta morbimortalidad de la patología vascular en pacientes intervenidos de urgencia.

La patología de columna se ubica en cuarto lugar, con $7 \%$ (5/72). La etiología más frecuente complicada fue el canal lumbar estrecho en $80 \%$ de los casos (4/5) y el restante $20 \%$ fue un caso con hernia discal lumbar. En esta patología no hubo muertes. Las intervenciones fueron todas de coordinación y solo el 20\% (1/5) tuvo evolución desfavorable mientras que el resto (4/5) evolucionó favorablemente $(80 \%)$.

Las complicaciones observadas fueron 6 en total, 4 infecciosas (67\%), un HED y una FLCR ( $16,5 \%$ cada una).

Las lesiones en nervios periféricos intervenidas se complicaron solamente 3\% (2/72), en ambas ocasiones de coordinación. La topografía fue el plexo braquial, en ambos casos vinculados a infección. Los dos pacientes presentaron una evolución favorable (mRS 0-2).

La patología infecciosa representó un solo caso (1\%) en una paciente de 30 años intervenida de urgencia por un absceso cerebral secundario a otitis media aguda (OMA) la cual falleció por la propia patología de base (grupo 2).

Los restantes diagnósticos (7/72) fueron agrupados de la siguiente manera: malformación de Chiari y defecto óseo, $43 \%$ cada uno (3/7). Se observó un solo trastorno circulatorio del líquido cefalorraquídeo (LCR) representado como hidrocefalia (14\%).
El paciente con defecto óseo intervenido de urgencia falleció de causa médica no determinada, por lo que la mortalidad específica de este subgrupo fue de 14\%. El resto evolucionó favorablemente.

Para el análisis de riesgo de muerte asociado al diagnóstico, tipo de complicación y oportunidad quirúrgica, se aplicó una regresión logística multivariada incluyendo todas las variables cuyo valor p no superó 0,25 en el análisis univariado. La oportunidad quirúrgica fue el único factor de riesgo significativo, considerando las covariables. La intervención de urgencia mostró 19 veces más riesgo de fallecer en un periodo postquirúrgico de 30 días con un nivel de significación de 0,018 (IC 95\% 1,657-208,732).

El análisis de $\mathrm{X}^{2}$ demostró una asociación entre diagnóstico vascular y morbimortalidad con un nivel de significación de $\mathrm{p}=0,015$, no así en los otros diagnósticos considerados. Las tablas de contingencia objetivaron asociación significativa entre presentar una complicación isquémica y morbimortalidad $(\mathrm{p}=0,024)$ y entre la presencia de HEC refractaria y outcome $(p=0,003)$.

Las demás complicaciones estudiadas y la morbimortalidad postquirúrgica se hallaron independientes entre sí. 
Tabla 5. Distribución y descripción de la población por patología según morbimortalidad específica.

\begin{tabular}{|c|c|c|c|c|c|c|c|c|c|c|c|c|}
\hline & \multicolumn{12}{|c|}{ Diagnósticos al ingreso $n=72$} \\
\hline & \multicolumn{3}{|c|}{$\operatorname{Vascular}(n=24)$} & \multicolumn{3}{|c|}{ Tumoral $(n=22)$} & \multicolumn{3}{|c|}{ Traumático $(n=11)$} & \multicolumn{3}{|c|}{ Columna $(n=5)$} \\
\hline & C & $U$ & $\%$ & C & $U$ & $\%$ & C & $U$ & $\%$ & C & $U$ & $\%$ \\
\hline Oportunidad quirúrgica & 4 & 20 & $(33,3 \%)$ & 21 & 1 & $(30,6 \%)$ & - & 11 & $(15,3 \%)$ & 5 & - & $(6,9 \%)$ \\
\hline \multicolumn{13}{|l|}{ Sexo } \\
\hline Masculino & 2 & 8 & $(41,7 \%)$ & 14 & - & $(63,6 \%)$ & - & 10 & $(90,9 \%)$ & 3 & - & $(60,0 \%)$ \\
\hline Femenino & 2 & 12 & $(58,3 \%)$ & 7 & 1 & $(36,4 \%)$ & - & 1 & $(9,1 \%)$ & 2 & - & $(40,0 \%)$ \\
\hline $\operatorname{Edad}(\overline{\mathbf{X}})$ & 48 & 51 & & 46 & 55 & & - & 34 & & 51 & - & \\
\hline \multicolumn{13}{|l|}{ Tipo de complicación } \\
\hline Hemorrágica & 2 & 2 & $(16,0 \%)$ & 7 & 1 & $(26,7 \%)$ & - & 3 & $(30,0 \%)$ & 1 & - & $(16,7 \%)$ \\
\hline Infecciosa & 1 & 11 & $(48,0 \%)$ & 10 & - & $(33,3 \%)$ & - & 5 & $(50,0 \%)$ & 4 & - & $(66,7 \%)$ \\
\hline Isquémica & 1 & 3 & $(16,0 \%)$ & 1 & - & $(3,3 \%)$ & - & - & & - & - & \\
\hline Hidrocefalia & - & 1 & $(4,0 \%)$ & 3 & - & $(10,0 \%)$ & - & - & & - & & \\
\hline Fístula LCR & 1 & - & $(4,0 \%)$ & 5 & - & $(16,7 \%)$ & - & 1 & $(10,0 \%)$ & 1 & - & $(16,7 \%)$ \\
\hline Otro & - & 3 & $(12,0 \%)$ & 3 & - & $(10,0 \%)$ & - & 1 & $(10,0 \%)$ & - & - & \\
\hline \multicolumn{13}{|l|}{ Presentación clínica } \\
\hline $\mathrm{HEC}$ & 4 & 18 & $(62,8 \%)$ & 12 & 1 & $(39,4 \%)$ & 11 & $(91,7 \%)$ & - & - & & \\
\hline SFN & 2 & 8 & $(28,6 \%)$ & 13 & - & $(39,4 \%)$ & 1 & $(9,1 \%)$ & - & - & & \\
\hline Sd. acromegálico & - & - & 3 & - & $(9,1 \%)$ & - & - & - & - & & & \\
\hline Sd. meníngeo & - & 3 & $(8,6 \%)$ & - & - & - & - & - & - & & & \\
\hline Sd. rrm & - & - & 2 & - & $(6,1 \%)$ & - & - & 5 & - & $(100 \%)$ & & \\
\hline Sd. cerebeloso & - & - & 2 & - & $(6,1 \%)$ & - & - & - & - & & & \\
\hline Hipotensión EC & - & - & - & - & - & - & - & - & & & & \\
\hline Defecto óseo & - & - & - & - & - & - & - & - & & & & \\
\hline \multicolumn{13}{|l|}{$m R S$} \\
\hline [0-2] Favorable & 3 & 4 & $(29,2 \%)$ & 13 & - & $(59,1 \%)$ & - & 5 & $(45,5 \%)$ & 4 & - & $(80,0 \%)$ \\
\hline$[3,5]$ Desfavorable & - & 3 & $(12,5 \%)$ & 4 & - & $(18,2 \%)$ & - & 1 & $(9,1 \%)$ & 1 & - & $(20,0 \%)$ \\
\hline [6] Mortalidad & 1 & 13 & $(58,3 \%)$ & 4 & 1 & $(22,7 \%)$ & - & 5 & $(45,5 \%)$ & - & - & \\
\hline \multicolumn{13}{|l|}{ Causa de muerte } \\
\hline Quirúrgica (grupo 1) & 1 & 2 & $(21,4 \%)$ & 3 & - & $(60,0 \%)$ & - & - & - & - & & \\
\hline Enfermedad (grupo 2) & - & 9 & $(64,3 \%)$ & - & 1 & $(20,0 \%)$ & - & 3 & $(60,0 \%)$ & - & - & \\
\hline Médica (grupo 3) & - & 2 & $(14,3 \%)$ & 1 & - & $(20,0 \%)$ & - & 2 & $(40,0 \%)$ & - & - & \\
\hline
\end{tabular}

C: coordinación; U: urgencias; LCR: líquido cefalorraquídeo; Sd.: síndrome; EC: endocraneana; SFN: FALTA; EC: endocraneana. 
Tabla 5. Distribución y descripción de la población por patología según morbimortalidad específica (continuación).

\begin{tabular}{|c|c|c|c|c|c|c|c|c|c|c|}
\hline \multirow[b]{3}{*}{ Oportunidad quirúrgica } & \multicolumn{10}{|c|}{ Diagnósticos al ingreso $n=72$} \\
\hline & \multicolumn{3}{|c|}{ Nervios periféricos $(n=2)$} & \multicolumn{3}{|c|}{ Infeccioso (n=1) } & \multicolumn{3}{|c|}{ Otros $(n=7)$} & \multirow[t]{2}{*}{ Total } \\
\hline & C & U & $\%$ & C & $U$ & $\%$ & C & $U$ & $\%$ & \\
\hline & 2 & & $(2,8 \%)$ & & 1 & $(1,4 \%)$ & 4 & 3 & $(9,7 \%)$ & $72(100 \%)$ \\
\hline \multicolumn{11}{|l|}{ Sexo } \\
\hline Masculino & 2 & - & $(100 \%)$ & - & - & & 2 & 2 & $(57,1 \%)$ & $43(59,7 \%)$ \\
\hline Femenino & - & - & & - & 1 & $(100 \%)$ & 2 & 1 & $(42,9 \%)$ & $29(40,3 \%)$ \\
\hline $\operatorname{Edad}(\bar{x})$ & 35 & - & & - & 30 & & 45 & 25 & & 45 \\
\hline \multicolumn{11}{|l|}{ Tipo de complicación } \\
\hline Hemorrágica & - & - & & - & - & & - & 1 & $(8,3 \%)$ & $17(19,8 \%)$ \\
\hline Infecciosa & 2 & - & $(100 \%)$ & - & - & & 2 & 4 & $(50,0 \%)$ & $39(45,3 \%)$ \\
\hline Isquémica & - & - & & - & - & & - & - & & $5(5,8 \%)$ \\
\hline Hidrocefalia & - & - & & - & - & & 1 & 2 & $(25,0 \%)$ & $7(8,1 \%)$ \\
\hline Fístula LCR & - & - & & - & - & & 2 & - & $(16,7 \%)$ & $10(11,6 \%)$ \\
\hline Otro & - & - & - & 1 & $(100 \%)$ & - & - & & & $8(9,3 \%)$ \\
\hline \multicolumn{11}{|l|}{ Presentación clínica } \\
\hline HEC & - & - & & - & 1 & $(100 \%)$ & 3 & 1 & $(44,4 \%)$ & $51(52,6 \%)$ \\
\hline SFN & 2 & & $(100 \%)$ & - & - & & 2 & - & $(22,2 \%)$ & $28(28,9 \%)$ \\
\hline Sd. acromegálico & - & - & & - & - & & - & - & & $3(3,1 \%)$ \\
\hline Sd. meníngeo & - & - & & - & - & & - & - & & $3(3,1 \%)$ \\
\hline Sd. RRM & - & - & & - & - & & - & - & & $7(7,2 \%)$ \\
\hline Sd. cerebeloso & - & - & & - & - & & - & - & & $2(2,1 \%)$ \\
\hline Hipotensión EC & - & - & & - & - & & - & 1 & $(11,1 \%)$ & $1(1,0 \%)$ \\
\hline Defecto óseo & - & - & & - & - & & 1 & 1 & $(22,2 \%)$ & $2(2,1 \%)$ \\
\hline \multicolumn{11}{|l|}{$m R S$} \\
\hline [0-2] Favorable & 2 & & $(100 \%)$ & - & - & & 4 & 2 & $(85,7 \%)$ & $37(51,4 \%)$ \\
\hline$[3,5]$ Desfavorable & - & - & & - & - & & - & - & & $9(12,5 \%)$ \\
\hline [6] Mortalidad & - & - & & 1 & - & $(100 \%)$ & - & 1 & $(14,3 \%)$ & $26(36,1 \%)$ \\
\hline \multicolumn{11}{|l|}{ Causa de muerte } \\
\hline Quirúrgica (grupo 1) & - & - & & - & - & & - & - & & $6(23,1 \%)$ \\
\hline Enfermedad (grupo 2) & - & - & & - & 1 & $(100 \%)$ & - & - & & $14(53,8 \%)$ \\
\hline Médica (grupo 3) & - & - & & - & - & & - & 1 & $(100 \%)$ & $6(23,1 \%)$ \\
\hline
\end{tabular}

C: Coordinación; U: Urgencias; LCR: líquido cefalorraquídeo; Sd.: síndrome; HEC: hipertensión endocraneana; SFN: FALTA; EC: endocraneana; RRM: raqui-radículo-medular. 
Tabla 6. Regresión logística multivariada - mortalidad.

\begin{tabular}{|c|c|c|c|c|c|c|c|c|}
\hline & OR crudo & Valor $p$ & B & SE & OR ajustado & Valor $p$ & $B$ & SE \\
\hline Tiempo quirúrgico & 0,7 & 0,024 & $-0,357$ & 0,158 & 0,819 & 0,308 & $-0,199$ & 0,195 \\
\hline \multicolumn{9}{|l|}{ Diagnóstico } \\
\hline Traumático & 1,587 & 0,486 & 0,462 & 0,663 & & & & \\
\hline Vascular & 4,2 & 0,007 & 1,435 & 0,532 & 2,096 & 0,323 & 0,74 & 0,323 \\
\hline Columna & 0 & 0,999 & $-20,823$ & $1.4210,36$ & & & & \\
\hline Tumoral & 0,416 & 0,164 & $-0,878$ & 0,631 & 10,878 & 0,076 & 2,387 & 0,076 \\
\hline Otro & 0,62 & 0,51 & $-0,479$ & 0,727 & & & & \\
\hline \multicolumn{9}{|l|}{ Complicación } \\
\hline Fístula LCR & 0 & 0,999 & $-20,797$ & $1.5191,52$ & & & & \\
\hline Hemorrágica & 0,579 & 0,396 & $-0,547$ & 0,644 & & & & \\
\hline Hidrocefalia & 0,328 & 0,32 & $-1,115$ & 1,124 & & & & \\
\hline Infecciosa & 0,529 & 0,219 & $-0,637$ & 0,518 & 0,584 & 0,409 & $-0,537$ & 0,409 \\
\hline Isquémica & 10,714 & 0,035 & 2,372 & 1,127 & 6,224 & 0,231 & 1,828 & 1,526 \\
\hline Otra & 2,87 & 0,266 & 1,054 & 0,948 & & & & \\
\hline Oportunidad quirúrgica & 8,68 & $<0,000$ & 2,161 & 0,589 & 18,599 & 0,018 & 2,923 & 1,234 \\
\hline
\end{tabular}

OR: odds ratio; B: coeficiente B; SE error estándar; LCR: líquido cefalorraquídeo.

\section{Discusión}

La búsqueda bibliográfica exhaustiva realizada objetivó tasas variadas de mortalidad global asociada a neurocirugía. Se encontraron 8 investigaciones internacionales con cifras de morbimortalidad global: Bydon (EE.UU.) reportó una tasa de $1,63 \%{ }^{(5)}$, Hammers (EE.UU.) reportó una tasa de $1,7 \%{ }^{(2)}$, Theodosopoulos (EE.UU.) reportó una tasa de $0,76 \%{ }^{(7)}$, Fuller (Uganda) informó cifras de $4 \%-6 \%{ }^{(6)}$. Brennan y colaboradores encontraron $3,7 \%$ de complicaciones en 30.121 pacientes $^{(10)}$. Otros reportes previos muestran una morbilidad relacionada a la cirugía de $16,4 \%$ a $28,3 \%{ }^{(11,12)}$.

El estudio de Hammers y colaboradores mostró con una mortalidad neuroquirúrgica global de $1,7 \%{ }^{(2)}$ donde la mayoría de las muertes se produjeron en pacientes que se sometieron a cirugías de urgencia.

Bydon y colegas evaluaron el impacto que tiene la participación de residentes en morbimortalidad asociada a neurocirugía usando la base de datos "The American College of Surgeons National Surgical Quality Improvement Program".

Encontraron que $15,8 \%$ de todos los pacientes neuroquirúrgicos incluidos en el estudio padeció por lo menos una complicación quirúrgica. La mortalidad total fue
$1,63 \%$. Otra investigación llevada a cabo en Uganda por Fuller demuestra una mortalidad neuroquirúrgica durante los años más recientes correspondiente a $4 \%-6 \%{ }^{(6)}$.

Theodosopoulos y colegas, en un estudio prospectivo multicéntrico, compilaron resultados clínicos de más de 5.000 procedimientos neuroquirúrgicos para evaluar estrategias de recolección de datos que mejor permitan obtener información precisa favoreciendo a su vez, el registro de datos conforme a la metodología establecida. El 95,1\% de los pacientes incluidos en el estudio no presentó complicaciones. Los autores de esta investigación observaron una mortalidad global de $0,76 \%$ y de $0,46 \%$ excluyendo intervenciones urgentes por hemorragia intracerebral no aneurismática ${ }^{(7)}$.

Kashiwasaki y colaboradores, en su servicio docente, encontraron una mortalidad de $2,1 \%$ y una morbilidad de $9,7 \%$ durante un periodo de 5 años. Ésta investigación demostró una clara mejoría de sus resultados neuroquirúrgicos al introducir lo que ellos denominan "conferencias de morbimortalidad", que constituyen el equivalente a los ateneos realizados en nuestro servicio ${ }^{(13)}$. 
No encontramos en la literatura un diseño metodológico que evaluara la morbimortalidad global y específica por patología en un mismo estudio.

Existen dos grandes problemas en Uruguay que dificultan el realizar un trabajo con estas características: el $\mathrm{N}$ de la población en estudio y la ausencia de una base de datos previa. Si bien la población total de estudio tiene un $\mathrm{N}$ de 477 pacientes en 2 años, lo cual es bajo comparado con otros países, logramos tener el $100 \%$ de la muestra y obtener así resultados que puedan ser comparables.

Del total de 72 pacientes operados que se complicaron, $65 \%$ (47/72) de ellos requirieron al menos una reintervención, lo que delata un porcentaje elevado. El promedio de edad del paciente complicado fue de 45 años, con una razón de 1,5/1 de leve predominio en el hombre (tabla 1).

La morbilidad global fue del $15 \%$, la mitad evolucionó favorablemente, $12,5 \%$ desfavorablemente y murió el $36 \%$ de los pacientes que se complicaron. La mortalidad global fue de 5,5\% en la población total, repartidos en $9,4 \%$ por la urgencia y $1,9 \%$ de coordinación (tabla 1). En comparación con estudios internacionales realizados en EE.UU. nuestras cifras son más altas, pero se asemejan a las observadas en Uganda $(4 \%-6 \%)^{(6)}$. En nuestra casuística vale la pena detenerse y observar que si bien el $36 \%$ de los pacientes que se complicaron luego murieron, el $81 \%$ eran operados de urgencia cuya mortalidad específica mostró que se trataba de pacientes graves y con altísimo riesgo de muerte. En ellos, la propia evolución de la enfermedad de base determinó el deceso en un $67 \%$ y el terreno biológico del paciente en un $24 \%$, es decir que la enfermedad de base y la condición médica impactaron negativamente, hecho que hace destacar la importancia de la continua profundización en el conocimiento y evolución natural de las diferentes patologías, así como también la correcta valoración del paciente que se opera de urgencia. En solo dos casos, 9\% restante de los fallecidos de urgencia, la causa estuvo directamente vinculada al acto quirúrgico, y en ambos casos se trataron de HSAe con aneurismas comunicantes anteriores operados por neurocirujano no subespecializado en cirugía vascular. De los pacientes fallecidos en coordinación (5/26), en el 80\% (4/5) la complicación deriva directamente del acto quirúrgico, mientras que en el restante $20 \%(1 / 5)$ fue vinculada a una causa médica. Este fenómeno es esperable dado que el paciente de coordinación es llevado en las mejores condiciones al acto quirúrgico y por tanto la propia evolución de la enfermedad no impacta tanto como en la urgencia de un paciente grave. Por tanto, si bien 4 casos en 254 operados de coordinación representa una mortalidad global del servicio de $1,6 \%$, cifra baja y similar a países de pri- mer mundo, la responsabilidad deriva en que estos 4 casos fueron complicaciones vinculadas al acto quirúrgico en sí. Tres de estos casos eran pacientes portadores de tumor cerebral que compartían un riesgo aumentado de sangrado por las características neoplásicas y el terreno previo de los pacientes. En 2 casos había una diseminación tumoral masiva de la enfermedad, con muy mal pronóstico y riesgo alto de sangrado. Y el tercer paciente falleció por una complicación vinculada a causa médica. Era un enfermo portador de un glioma de alto grado ingresado para cirugía de coordinación, que 48 horas después de la cirugía de urgencia murió por sepsis respiratoria. En retrospectiva se evidenció una pancitopenia y sepsis respiratoria como causa de su deterioro de consciencia y no la agravación del tumor. En estos pacientes neoplásicos la exhaustiva valoración previa y la selección más minuciosa de la indicación quirúrgica podría haber previsto y evitado la cirugía. En el caso de la patología vascular es imperativo que aquellos aneurismas de complejidad sean resueltos por el neurocirujano vascular subespecializado y formado en dicha patología, tal y como lo expresan las distintas guías mundiales ${ }^{(8)}$. Cuando analizamos la morbimortalidad específica, podemos saber que patología neuroquirúrgica tuvo más complicaciones y muertes y como evolucionaron en el mes postoperatorio, lo cual nos permite ver el comportamiento por subespecialidad (tabla 5).

En ese sentido la patología vascular fue la entidad que más complicó ( $1 / 3$ de los casos), dominada por la naturaleza hemorrágica y dentro de ésta la HSAe por rotura de aneurisma fue la predominante por lejos (tabla 1). Hay una clara diferencia en aquellos pacientes que se operaron de coordinación y urgencia. El único paciente vascular operado de coordinación que murió era portador de un aneurisma comunicante posterior complejo donde de forma inadvertida se clipó la arteria carótida interna luego de presentar una rotura aneurismática intraoperatoria, lo que representa una mortalidad global en patología vascular de coordinación de 1,4\% (1/69). Las mismas consideraciones son advertidas en este caso, este aneurisma complejo fue operado por un neurocirujano no subespecializado en cirugía vascular. En la urgencia, la mortalidad global de la patología representó $19 \%(13 / 69)$ de la población total y la evolución de los pacientes fue favorable solo en $20 \%$, hecho que se explica por los factores analizados anteriormente, terreno biológico malo y gravedad inicial (tablas 1,2 y 5 ). La morbilidad quirúrgica global en la patología vascular fue de $29 \%$ (20/69) en la urgencia y 4,3\% (3/69) de coordinación. En cuanto a la patología tumoral encontramos una mortalidad de $4,5 \%$ (tabla 1 ). Hubo un caso que falleció de urgencia debido a que presentaba una sepsis respiratoria y los otros tres casos, cuya complicación de- 
rivó del acto quirúrgico fueron debido a complicaciones hemorrágicas y otro por su enfermedad de base. En el paciente que presentaba la sepsis respiratoria hubo un error en la interpretación de la clínica y no se sospechó dicha complicación. La patología traumática tuvo una mortalidad de $5 \%$ y una morbilidad de $9,8 \%$, destacando que no hubo ninguna complicación derivada del acto quirúrgico.

En la patología de columna no hubo fallecidos y la gran mayoría presentó una evolución favorable. Se trata de una patología corriente en la práctica neuroquirúrgica, con bajo riesgo de complicaciones.

\section{Fortalezas y debilidades del trabajo}

El $\mathrm{N}$ bajo es la gran debilidad, pero de todas formas representa la morbilidad y mortalidad de nuestra población intervenida. La gran fortaleza es el impacto que esto tiene en poner en marcha estrategias en pos de la mejoría en las complicaciones y muerte, ya sea mediante la formación y capacitación de los profesionales, insistir en la correcta valoración del paciente para minimizar los riesgos de complicaciones, tener un referente en cada subespecialidad lo cual permite mejorar los resultados por tener una mayor formación en esa patología. Dada la asociación observada entre ciertas complicaciones y morbimortalidad, se rechaza la hipótesis nula con un nivel de significación de 0,024 (tabla 6). Por lo tanto, la evidencia sugiere que padecer una complicación isquémica o HEC postquirúrgica repercute en la morbimortalidad del paciente. No se esperaba identificar una asociación entre presentar un diagnóstico de tipo vascular y la morbimortalidad resultante, cuyo análisis merece la misma interpretación, la resolución de patología vascular compleja en manos no subespecializadas.

Era de esperar una asociación entre oportunidad quirúrgica y mortalidad, dada la evidencia proporcionada por investigaciones internacionales. Hammers y colaboradores demostraron una relación no significativa entre la cantidad de consultas en departamentos de emergencia y mayor mortalidad y observaron que la mayoría de las muertes se produjeron en pacientes que se sometieron a cirugías no coordinadas $^{(2)}$. Estos hechos, si bien constituyen signos indirectos del riesgo que conlleva someterse a una intervención urgente, concuerdan con los hallazgos de la presente investigación: que las intervenciones neuroquirúrgicas urgentes impactan en una mayor mortalidad.

Los autores introducen y definen tres grupos de pacientes para estudiar el vínculo entre causa de muerte y mortalidad lo cuál tiene una enorme importancia en poder estudiar el vínculo en pos de una mejora en los resultados.

\section{Conclusiones y perspectivas}

Por primera vez se realiza en nuestro país un estudio de morbimortalidad asociada a la patología neuroquirúrgica, no existiendo en la literatura mundial análisis de morbimortalidad general y específica en un único diseño. A partir de aquí sabemos con datos objetivos cuál es la morbilidad y mortalidad en nuestro centro, y comenzar a trabajar sobre una estructura sólida para disminuir aún más estos indicadores de salud en mayores investigaciones a futuro. El vínculo entre la causa de muerte y mortalidad fue dominada por el grupo 2 y 3 en la neurocirugía de urgencia, mientras que el grupo 1 fue el gran responsable de las muertes y resultados desfavorables en la coordinación. En este sentido la selección y valoración integral del paciente, así como también la formación del profesional y subespecialización son factores netamente influyentes en las muertes y resultados desfavorables. Dentro de las subespecialidades, la neurocirugía vascular dejó en evidencia este aspecto, fenómeno que ocurre por la alta complejidad de la patología, con poco margen al error. Los factores diagnóstico vascular, complicación isquémica y HEC surgen como elementos influyentes en la evolución postquirúrgica desde una perspectiva general, así como también la oportunidad quirúrgica. Si bien la morbimortalidad global y específica es aceptable, sabemos que puede descender mucho más corrigiendo los factores mencionados. Se hace evidente la necesidad de crear una base de datos para explorar los posibles influyentes en morbimortalidad neuroquirúrgica con mayor profundidad y mayor cantidad de observaciones, con el fin de dilucidar las circunstancias que repercuten en los resultados clínicos.

\section{Agradecimientos}

Agradecemos al Dpto. de Métodos Cuantitativos de la Facultad de Medicina, UdelaR, por sus aportes y correcciones.

Al todo el Staff del Dpto. de Neurocirugía del Hospital de Clìnicas, docentes, residentes y archivos médicos.

Y especialmente al Dr. Abayubá Perna y Dr. Sergio Mele por sus correcciones en la introducción de datos al Epidata y análisis estadístico.

\section{Abstract}

Introduction: morbidity and mortality rates allow for the evaluation of the quality of care and outcome and they also make it possible to compare different healthcare centers. This is the first morbidity and mortality study in neurosurgery carried out in Uruguay.

Objective: to determine the global and specific morbidity and mortality rates in the Neurosurgery Department at the Clínicas University Hospital, and to determi- 
ne if mortality and morbidity are associated to surgical complications.

Method: retrospective, longitudinal, observational and descriptive analysis of all patients $\geq 15$ years old that underwent a neurosurgical procedure between April 2017 and 2019. Data were obtained from patient medical records. The following variables were analyzed: age, sex, comorbidity, clinical data, diagnosis, opportunity of surgical procedure, complications, type of complication, mortality, cause of mortality, outcome and surgical time.

Results: 477 patients underwent neurosurgical procedure, 72 of which were complicated surgeries. Overall mortality was 5.5\% (26/477) and morbidity $15 \%$ (72/477). 36\% of complicated patients died (26/72). Vascular pathology was the specific morbidity that complicated patients the most $20 \%$ (14/69). Infection was the most frequent type of complication $46 \%$ $(39 / 84)$. The evolution of the disease itself and the terrain caused the death of $90 \%$ of complicated patients undergoing emergency surgery (19/21), being the latter an independent risk factor for death $(\mathrm{p}=0.018)$.

As to coordinated surgeries, the cause of death was associated to the surgical act (80\%). Association was found between vascular pathology and morbidity and mortality $(\mathrm{p}=0.015)$ and between ischemic complication and morbidity and mortality $(\mathrm{p}=0.024)$. The presence of intracranial hypertension $(\mathrm{IH})$ was associated with a bad outcome $(\mathrm{p}=0.003)$.

Conclusions: the results show a good quality of care compared to other centers. There are still aspects to correct to reduce morbidity and mortality rates.

\section{Resumo}

Introdução: a análise da morbimortalidade permite avaliar a qualidade do atendimento e dos resultados e comparar diferentes centros de saúde. Este é o primeiro estudo de morbimortalidade em neurocirurgia realizado no Uruguai.

Objetivo: determinar a morbimortalidade global e específica no Departamento de Neurocirurgia do Hospital de Clínicas e a associação entre complicação e morbimortalidade.

Metodologia: estudo observacional, descritivo-analítico, longitudinal, retrospectivo de todos os pacientes $\geq 15$ anos que necessitaram de cirurgia entre abril de 2017 e 2019. Os dados foram obtidos dos prontuários dos pacientes e as variáveis analisadas foram: idade, sexo, comorbidade, aspectos clínicos, diagnóstico, oportunidade cirúrgica, complicação, tipo de complicação, mortalidade, causa da mortalidade, resultado e tempo cirúrgico.
Resultados: 477 pacientes foram operados dos quais 72 apresentaram complicações. A mortalidade geral foi de 5,5\% (26/477) e a morbidade de $15 \%$ (72/477). $36 \%$ dos pacientes com complicações morreram $(26 / 72)$.

A patologia vascular foi a causa específica de mortalidademorbidade específica mais freqüente $20 \%$ (14/69). A infecção foi o tipo de complicação mais freqüente $46 \%$ (39/84). A evolução da própria doença e do local da cirurgia ocasionou a morte de $90 \%$ dos pacientes com complicações submetidas a cirurgias de urgência (19/21), sendo este último fator de risco independente para óbito $(\mathrm{p}=0,018)$.

Nas cirurgias eletivas, a causa da morte esteve ligada ao ato cirúrgico $(80 \%)$. Houve associação entre patologia vascular e morbimortalidade $(p=0,015)$ e entre complicação isquêmica e morbimortalidade $(p=0,024)$. A presença de hipertensão intracraniana (HEC) foi associada a um desfecho ruim $(\mathrm{p}=0,003)$.

Conclusões: os resultados mostram uma boa qualidade de atendimento em comparação com outros centros. Ainda há aspectos a serem corrigidos para reduzir a morbimortalidade.

\section{Referencias bibliográficas}

1. Lassen B, Helseth E, Rønning P, Scheie D, Johannesen TB, Mæhlen J, et al. Surgical mortality at 30 days and complications leading to recraniotomy in 2630 consecutive craniotomies for intracranial tumors. Neurosurgery 2011; 68(5):1259-68.

2. Hammers R, Anzalone S, Sinacore J, Origitano T. Neurosurgical mortality rates: what variables affect mortality within a single institution and within a national database?. J Neurosurg 2010; 112(2):257-64.

3. Campbell E, Beez T, Todd L. Prospective review of 30-day morbidity and mortality in a paediatric neurosurgical unit. Childs Nerv Syst 2017; 33(3):483-9.

4. Saver JL, Filip B, Hamilton S, Yanes A, Craig S, Cho M, et al. Improving the reliability of stroke disability grading in clinical trials and clinical practice: the Rankin Focused Assessment (RFA). Stroke 2010; 41(5):992-5.

5. Bydon M, Abt N, De la Garza-Ramos R, Macki M, Witham T, Gokaslan Z, et al. Impact of resident participation on morbidity and mortality in neurosurgical procedures: an analysis of 16,098 patients. J Neurosurg 2015; 122(4): 955-61.

6. Fuller AT. Neurosurgical outcomes following establishment of a twinning program at Mulago Hospital in Uganda [Tesis de Maestría]. Durham: Global Health Institute in the Graduate School of Duke University, 2015.

7. Theodosopoulos PV, Ringer AJ, McPherson CM, Warnick RE, Kuntz C 4th, Zuccarello M, et al. Measuring surgical outcomes in neurosurgery: implementation, analysis, 
and auditing a prospective series of more than 5000 procedures. J Neurosurg 2012; 117(5):947-54.

8. Bederson JB, Connolly ES Jr, Batjer HH, Dacey RG, Dion JE, Diringer MN, et al. Guidelines for the management of aneurysmal subarachnoid hemorrhage: a statement for healthcare professionals from a special writing group of the Stroke Council, American Heart Association. Stroke 2009; 40:994-1025.

9. Broggi M, Zattra C, Ferroli P. How to compare outcomes and complications in neurosurgery: we must make the mission possible! Surg Neurol Int 2018; 9(1):65.

10. Brennan TA, Leape LL, Laird NM, Hebert L, Localio AR, Lawthers AG, et al. Incidence of adverse events and negli- gence in hospitalized patients. Results of the Harvard Medical Practice Study I. N Engl J Med 1991; 324(6):370-6.

11. Bonsanto MM, Hamer J, Tronnier V, Kunze S. A complication conference for internal quality control at the Neurosurgical Department of the University of Heidelberg. Acta Neurochir Suppl 2001; 78:139-45.

12. Houkin K, Baba T, Minamida Y, Nonaka T, Koyanagi I, Iiboshi S. Quantitative analysis of adverse events in neurosurgery. Neurosurgery 2009; 65(3):587-94.

13. Kashiwazaki D, Saito H, Uchino H, Akioka N, Hori E, Shibata $\mathrm{T}$, et al. Morbidity and mortality conference can reduce avoidable morbidity in neurosurgery: its educational effect on residents and surgical safety outcomes. World Neurosurg 2020; 133:e348-e355.

\footnotetext{
Contribución de autores

Rodrigo Moragues, ORCID 0000-0002-3739-183X. Concepción, diseño, revisión crítica.

Gonzalo Bertullo, ORCID 0000-0002-3171-7915. Concepción, diseño, ejecución, análisis, interpretación de los resultados, redacción, revisión crítica.

Víctor Reyes, ORCID 0000-0002-5172-2447. Concepción, diseño, ejecución, análisis, interpretación de los resultados, redacción, revisión crítica.

Laura Lanning, ORCID 0000-0002-0615-3505. Concepción, diseño, ejecución, análisis, interpretación de los resultados, redacción, revisión crítica.

Agustín Olivera, ORCID 0000-0001-9602-3175. Concepción, diseño, ejecución, análisis, interpretación de los resultados, redacción, revisión crítica.

Rodrigo Veiga, ORCID 0000-0003-4394-1436. Concepción, diseño, ejecución.

Cristian Cardozo, ORCID: 0000-0003-0502-4012. Concepción, diseño, ejecución.

Patricia Álvarez, ORCID 0000-0002-3373-4284. Participación: Concepción, diseño, ejecución.
} 